\title{
Characterization of Ablation Thresholds for 3D-Cultured Patient-Derived Glioma Stem Cells in Response to High-Frequency Irreversible Electroporation
}

\author{
J. W. Ivey ${ }^{1}$, E. M. Wasson ${ }^{2}$, N. Alinezhadbalalami ${ }^{1}$, A. Kanitkar ${ }^{1}$, W. Debinski ${ }^{3}$, Z. Sheng ${ }^{4,5,6}$, \\ R. V. Davalos ${ }^{1,2,3,6}$, and S. S. Verbridge ${ }^{1,2,3,6, \star}$ \\ ${ }^{1}$ School of Biomedical Engineering and Sciences, Virginia Tech-Wake Forest University, Blacksburg, VA 24061, USA \\ ${ }^{2}$ Department of Mechanical Engineering, Virginia Tech, Blacksburg, VA 24061, USA \\ ${ }^{3}$ Brain Tumor Center of Excellence, Comprehensive Cancer Center, Wake Forest Baptist Medical Center, Winston-Salem, \\ NC 27157, USA \\ ${ }^{4}$ Virginia Tech Carilion Research Institute, Roanoke, VA 24061, USA \\ ${ }^{5}$ Department of Internal Medicine, Virginia Tech Carilion School of Medicine, Roanoke, VA 24016, USA \\ ${ }^{6}$ Faculty of Health Science, Virginia Tech, Blacksburg, VA 24061, USA \\ ${ }^{\star}$ Correspondence should be addressed to S. S. Verbridge; sverb@vt.edu
}

Received 20 July 2018; Accepted 18 March 2019; Published 28 April 2019

Copyright (c) 2019 J. W. Ivey et al. Exclusive Licensee Science and Technology Review Publishing House. Distributed under a Creative Commons Attribution License (CC BY 4.0).

\begin{abstract}
High-frequency irreversible electroporation (H-FIRE) is a technique that uses pulsed electric fields that have been shown to ablate malignant cells. In order to evaluate the clinical potential of H-FIRE to treat glioblastoma (GBM), a primary brain tumor, we have studied the effects of high-frequency waveforms on therapy-resistant glioma stem-like cell (GSC) populations. We demonstrate that patient-derived GSCs are more susceptible to H-FIRE damage than primary normal astrocytes. This selectivity presents an opportunity for a degree of malignant cell targeting as bulk tumor cells and tumor stem cells are seen to exhibit similar lethal electric field thresholds, significantly lower than that of healthy astrocytes. However, neural stem cell (NSC) populations also exhibit a similar sensitivity to these pulses. This observation may suggest that different considerations be taken when applying these therapies in younger versus older patients, where the importance of preserving NSC populations may impose different restrictions on use. We also demonstrate variability in threshold among the three patient-derived GSC lines studied, suggesting the need for personalized cell-specific characterization in the development of potential clinical procedures. Future work may provide further useful insights regarding this patient-dependent variability observed that could inform targeted and personalized treatment.
\end{abstract}

\section{Introduction}

Glioblastoma (GBM), the most common and deadly primary brain tumor, has a dismal prognosis that has remained relatively unchanged despite decades of research [1]. A GBM tumor proves fatal within about 14 months even with multimodal intervention [2]. GBM tumors are treated with surgery followed by concurrent radiotherapy and adjuvant chemotherapy [3-5]. Neither single therapies nor treatments used in combination are curative and they are often debilitating to the patient. The failure of current treatments to greatly extend life expectancy is attributable, among other reasons, to several classes of therapy-resistant cells that propel tumor recurrence, which is nearly universal with GBM [6]. There exists a real need for next-generation GBM therapies, for use alone or in combination with current therapies, which can target the resistant cell populations and prevent tumor recurrence.

The highly therapy-resistant nature of GBM is due in large part to inter- and intratumor heterogeneity [7-12], which becomes a survival advantage for the tumor in resisting treatment $[13,14]$. In addition, presence of blood brain barrier contributes to failure of most chemotherapies by preventing most therapeutic regents from penetrating into the tumor. Central to the highly heterogeneous makeup of a GBM tumor is its initiator cells that are the progenitors from which the 
many subclasses of cells that make up a tumor are derived. It has been hypothesized that just as an organ develops from stem cells, tumors such as GBM are similarly derived from a set of stem-like cells that make up a small percentage of the tumor but drive its development and progression [15]. There is still some controversy over whether these cancer stem cells are originator cells, responsible for the initiation and progression of the tumor or whether they are a product of tumor initiation and evolution [16]. However, regardless of their standing in the hierarchy of the tumor, they possess two characteristics that make them very important in the study of cancer therapies-their ability to self-renew and initiate new tumors and their ability to resist current cancer therapies. What have come to be known as glioma stemlike cells (GSCs) or brain tumor initiating cells (BTICs) are a class of cells in the brain that express high levels of stem cell markers involved in self-renewal as well as genes involved in neural stem cell (NSC) proliferation and differentiation.

In addition to their self-renewal properties, cancer stem cells have another important characteristic central to their role in the tumor hierarchy, that is, their high degree of resistance and hyperactive repair mechanisms. GSCs have been shown to have a variety of resistance mechanisms such as high expression levels of a variety of drug resistance genes (BCRP, MDR1). GSCs additionally show enhanced DNA repair capacities, linked to increased MGMT activity, increased expression of damage checkpoints, and highly activated apoptosis inhibitors [17-21]. Multiple molecular mechanisms have been identified in GSCs to mediate therapeutic resistance to cytotoxic therapies such as Notch [22], NF- $\kappa$ B [23], EZH2 [24], and PARP [25]. Additional mechanisms of resistance may evolve from exposure to microenvironmental factors such as hypoxia [26, 27] and metabolic stress [28, 29]. These cells are able to maintain a tumor despite multimodal assaults by chemotherapies and radiation therapies [30, 31]. In general, cells are characterized as GSCs based on five criteria-ability for self-renewal, differentiation potential, high tumorigenicity, drug resistance, and radio-resistance $[32,33]$. Together, these features of GSCs make them highly likely to significantly contribute to GBM recurrence and therefore a very attractive therapeutic target [34-36].

Because many methods of cancer treatment are ineffective for GSCs and because they are important in tumor progression and recurrence, a need exists for a different class of therapies that can work effectively against GSCs. Conventional therapy regimes often eliminate the bulk tumor while unintentionally concentrating the pool of GSCs that can propagate a tumor, resulting in near universal recurrence in GBM tumors [37]. We previously hypothesized that irreversible electroporation (IRE) is a therapy that holds promise for the targeting of GSCs. However, this hypothesis has not yet been directly tested for patient-derived GSCs that are highly relevant in human GBM progression and recurrence.

IRE is a nonthermal ablation mechanism used primarily for the treatment of surgically inoperable tumors [38-40]. In IRE treatment, two or more electrodes are inserted into the tumor and short $(\sim 100 \mu \mathrm{s})$ high intensity monopolar pulses are applied to the tumor region [41]. These pulsed electric fields (PEFs) cause destabilization of the cell membrane which leads to cell death through loss of homeostasis [42$45]$. A second generation of IRE uses shorter $(\sim 0.5-2 \mu \mathrm{s})$ bipolar pulses delivered as a series of bursts. This treatment is known as high-frequency irreversible electroporation $(\mathrm{H}-$ FIRE). This therapy was developed due to its ability to reduce muscle contractions induced by IRE [46] and has been shown to elicit a more predictable response in heterogeneous tissue [47]. In addition, our previous studies have shown that by using H-FIRE; the efficacy of the treatment is enhanced in some cases for cells with a higher nuclear-to-cytoplasm ratio $[48,49]$. It has also been shown that IRE and $\mathrm{H}$ FIRE facilitate temporary disruption of blood brain barrier, allowing for penetration of therapeutic reagents [50, 51]. Disruption of blood brain barrier can be expected at the site of treatment, as well as in the sublethal surroundings, enabling the local delivery of drugs to the tumor site. Such combinatory treatments can provide a synergistic effect on eradicating the malignant cells.

Previous studies of the structural characterization of GSCs have reported atypical and enlarged nuclei in GSCs as well as irregular physical structure in other organelles [32, 52, 53]. Because we have previously demonstrated that nuclear size is strongly correlated with H-FIRE cell damage, we further hypothesize that not only will GSCs be susceptible to H-FIRE damage, but also this susceptibility may be enhanced compared with normal cell types such as astrocytes, which possess normal nuclear size. In this study, we therefore seek to determine the ability of H-FIRE therapy to ablate GSCs and the possibility of selective targeting of these cells. Such selectivity may be due to structural differences such as nuclear size or nuclear-to-cytoplasm ration (NCR), as well as yetto-be discovered mechanistic differences in H-FIRE cellular responses.

\section{Results}

2.1. GSCs Exhibit Tumor-Characteristic Enlarged Nuclei Compared to Normal Astrocytes. The GSCs tested all grow as nonadherent tumorspheres, a common morphological characteristic of stem cells. To confirm that GSCs follow the trend of enlarged nuclear-to-cytoplasm ratio (NCR) that many other cancerous cells have been shown to exhibit, we used confocal imaging to determine the nuclear and cell size of GSCs in three-dimensional type 1 collagen hydrogels. These measurements were compared to similar measurements taken of established differentiated (bulk) U-251 MG GBM cells and nonmalignant human astrocytes (NHAs) as well as nonmalignant human neural stem cells (NSCs) (Figure 1).

As seen in Figure 1(b), within the 3D collagen hydrogels the nuclear sizes of the GSC populations (GBM10, VTC-061, and VTC-064) and U-251 GBM cells are significantly larger than the healthy NHAs. The GSCs have a nuclear size similar to those of U251 bulk tumor cells. Interestingly nonmalignant NSCs exhibit cell morphologies similar to malignant GSCs with nuclear areas larger than healthy astrocytes. Because of their spherical shape, the GSCs and NSCs both have substantially less spreading and therefore a smaller cytoplasmic 

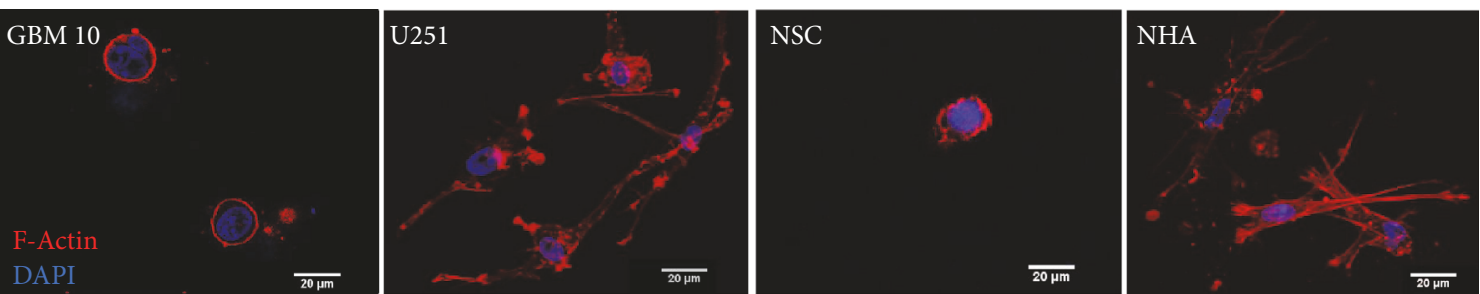

(a)

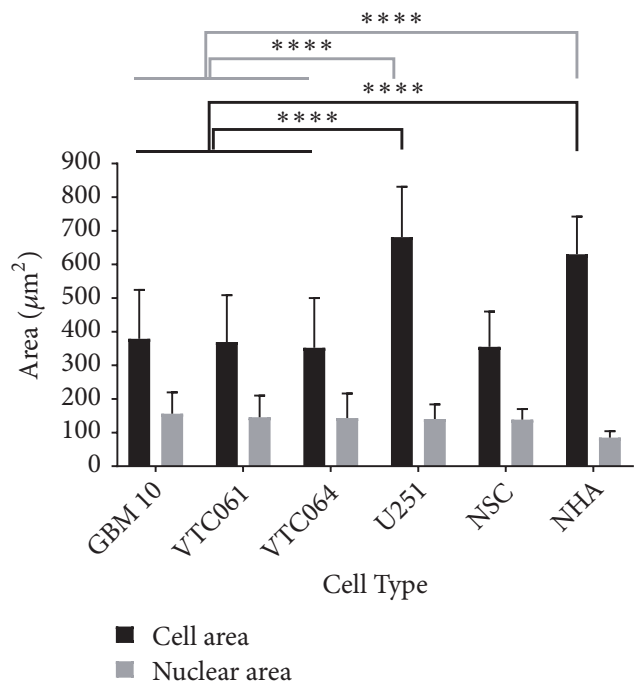

(b)

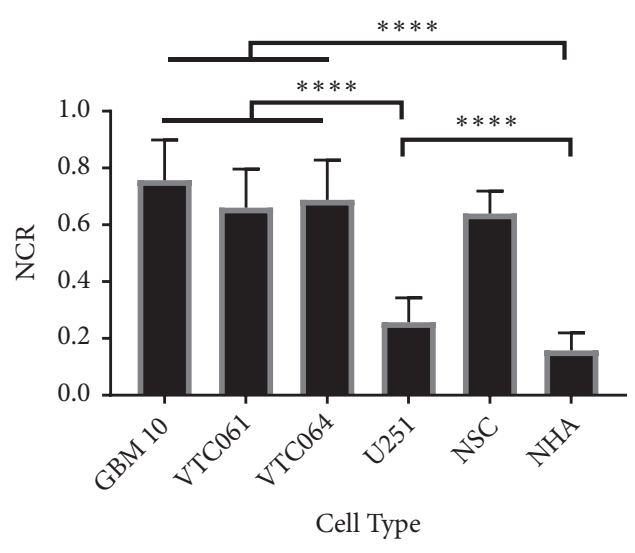

(c)

FIGURE 1: Cell morphology characterization in collagen hydrogels. (a) Confocal images of GSCs, U251, NSCs, and NHAs show differences in cell morphology of different cell types. (b) Cell areas for GSCs and NSCs are significantly smaller than U251 or NHAs ( $p<0.0001$ ). Nuclear areas for GSCs, U251 cells, and NSCs are enlarged compared to NHAs ( $p<0.0001)$. (c) Calculation of NCR from confocal images shows a significantly higher NCR for GSC and NSC populations compared to both U251 and NHA ( $p<0.0001)$. U251 have significantly higher NCRs than NHAs $\left({ }^{* * * *} \mathrm{p}<0.0001\right)$. (d) A comparison of circularity and aspect ratio across cell types illustrates the different cell morphology of GSCs and NSCs compared to U251 and NHA cells. GSCs and NSCs have a significantly higher circularity $\left({ }^{* * * *} \mathrm{p}<0.0001\right)$ than U251s and NHAs and a significantly lower aspect ratio $\left({ }^{* * * *} \mathrm{p}<0.0001\right)$. U251s and NHA have no significant difference in circularity or aspect ratio ( $\mathrm{p}$ $>0.05)$.

area than bulk tumor cells or astrocytes. This morphological feature is reflected in their nuclear-to-cytoplasm ratio, which is significantly greater for GSCs and NSCs than that of either bulk tumor cells or healthy cells (Figure 1(c)).

\subsection{GSCs Are Variably Sensitive to H-FIRE-Induced Cell} Death. Three different populations of patient-derived GSCs (GBM 10, VTC-061, and VTC-064) were seeded in 3D collagen hydrogels and exposed to H-FIRE therapy with pulse waveforms of $0.5 \mu \mathrm{s}-2 \mu \mathrm{s}-0.5 \mu \mathrm{s}$ (Figure 7). For all GSC populations an ablation zone (lesion) was produced by exposing the hydrogel to H-FIRE pulses (Figure 2(a)). To understand the effect of H-FIRE on GSC populations, these ablation areas were compared to the ablation areas for U251 differentiated tumor cells, NHAs, and NSCs. The ablation areas of all three GSC populations tested were significantly greater than the ablation area of NHA-seeded hydrogels ( $\mathrm{p}$ $<0.0001$ ) (Figure 2(b)). Only one GSC population, GBM 10, had a significantly larger ablation area than U-251 established GBM cells $(p=0.0002)$. GBM-10 cells also had a significantly greater ablation area than the NSCs $(p=0.0002)$. Yet the
NSCs had an ablation area similar to the other malignant cell types, significantly greater than the ablation area for NHAs. The GBM-10 cell population had significantly greater ablation area than VTC-061 ( $<<0.0001)$ and VTC-064 (p=0.0012) as well.

As seen in Figure 2(c), the larger ablation areas of GSCs, U-251s, and NSCs correspond to lower lethal electric field thresholds compared to healthy astrocytes. For the GBM10 population treated with $\mathrm{H}$-FIRE delivering a $0.5 \mu \mathrm{s}-2 \mu \mathrm{s}-$ $0.5 \mu \mathrm{s}$ waveform, the lethal threshold is $816 \pm 84 \mathrm{~V} / \mathrm{cm}$. For VTC-061 cells, the lethal threshold is $1019 \pm 70 \mathrm{~V} / \mathrm{cm}$. For VTC-064 cells the lethal threshold is $949 \pm 73 \mathrm{~V} / \mathrm{cm}$. The lethal threshold for the GBM-10 cell population is significantly lower than the lethal threshold for U-251s which have a lethal threshold of $1020 \pm 120 \mathrm{~V} / \mathrm{cm}(\mathrm{p}=0.0002)$. The VTC-061 and VTC-064 GSC populations treated with a $0.5 \mu \mathrm{s}-2 \mu \mathrm{s}-0.5 \mu \mathrm{s}$ waveform are not significantly different than U-251 bulk cells nor the NSCs. The NSCs had a lethal threshold of $1015 \pm 99 \mathrm{~V} / \mathrm{cm}$, similar to the bulk tumor cells and the VTC-064 and VTC-061 cell populations. The lethal threshold for NHA is significantly greater than the 


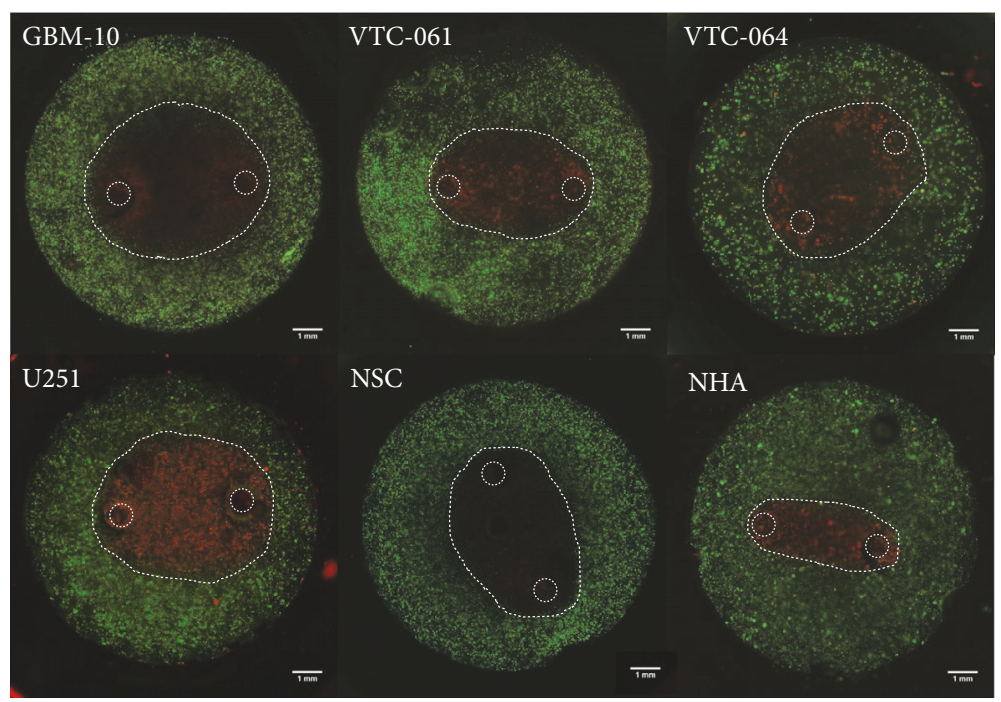

(a)

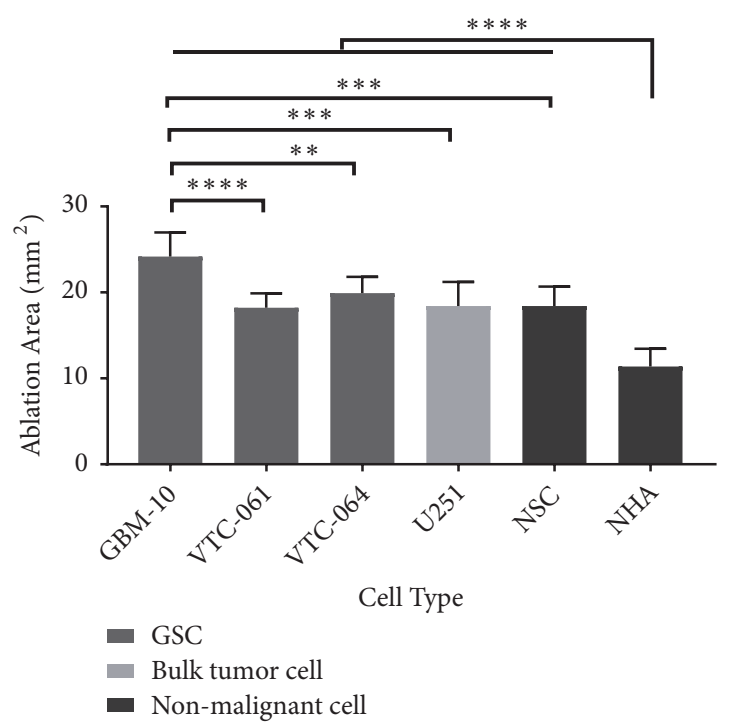

(b)

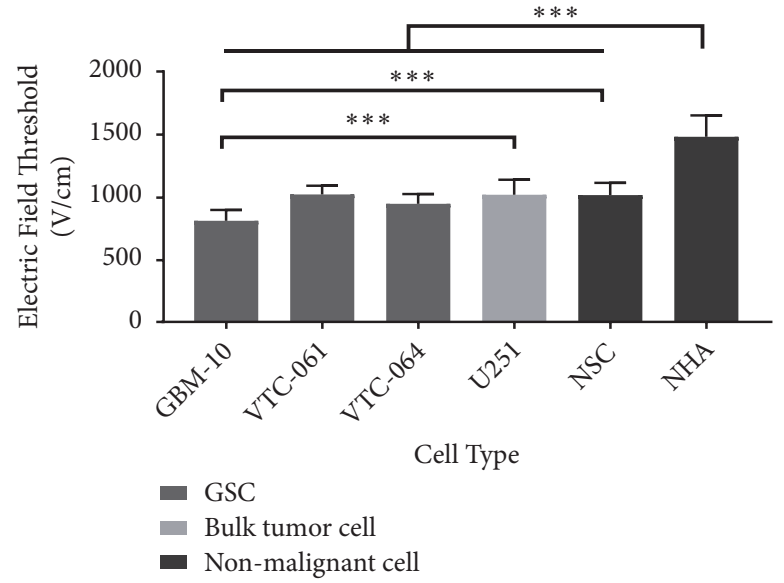

(c)

FIGURE 2: Populations of patient-derived glioma stem cells are responsive to H-FIRE therapy at variable but lower thresholds than healthy astrocytes. (a) A visible lesion was created in collagen hydrogels seeded with cells. Electrode placement and ablation lesion outlined by dotted white line (b) Comparison of lesion areas shows three GSC populations have greater lesion sizes than healthy astrocytes and similar lesion sizes to bulk tumor cells and NSCs (c) GSC populations have a lower lethal threshold than healthy astrocytes and similar thresholds to U251 cells and NSCs when exposed to H-FIRE pulses. ${ }^{* *} \mathrm{p}<0.01,{ }^{* * *} \mathrm{p}<0.001$, and ${ }^{* * * *} \mathrm{p}<0.0001$.

thresholds for the GSCs, NSCs, and U251s at $1480 \pm 175 \mathrm{~V} / \mathrm{cm}$ $(\mathrm{p}<0.0001)$.

Because GSCs grow in tumorspheres that can reach hundreds of cells in size, we next determined the lethal threshold of cells in such large tumorspheres to determine if the bunched cell morphology changes response to H-FIRE. GSCs were cultured in hydrogels for 9 days and exposed to $\mathrm{H}-$ FIRE pulses. This time-scale allowed cells to grow into large neurospheres within the collagen hydrogels. The spheroid clusters exhibit no significant difference in H-FIRE ablation area from single cell GSC hydrogels (Figure 3). From these results it can be concluded that the growth of the studied cells in 3D-spheroids does not affect GSC response to H-FIRE treatment.

2.3. Hyaluronic Acid Scaffolds Induce an Altered Cell Morphology. We next conducted control experiments with the goal of providing a more brain-relevant extracellular matrix (ECM) environment. To accomplish this, we seeded each representative cell type-GSC, bulk tumor cell, and nonmalignant astrocyte-into hydrogels composed of hyaluronic acid (HA). HA is a major component of brain ECM and is upregulated in GBM tumors [54, 55]. HA is important in maintaining the stem cell phenotype of GSCs and has 


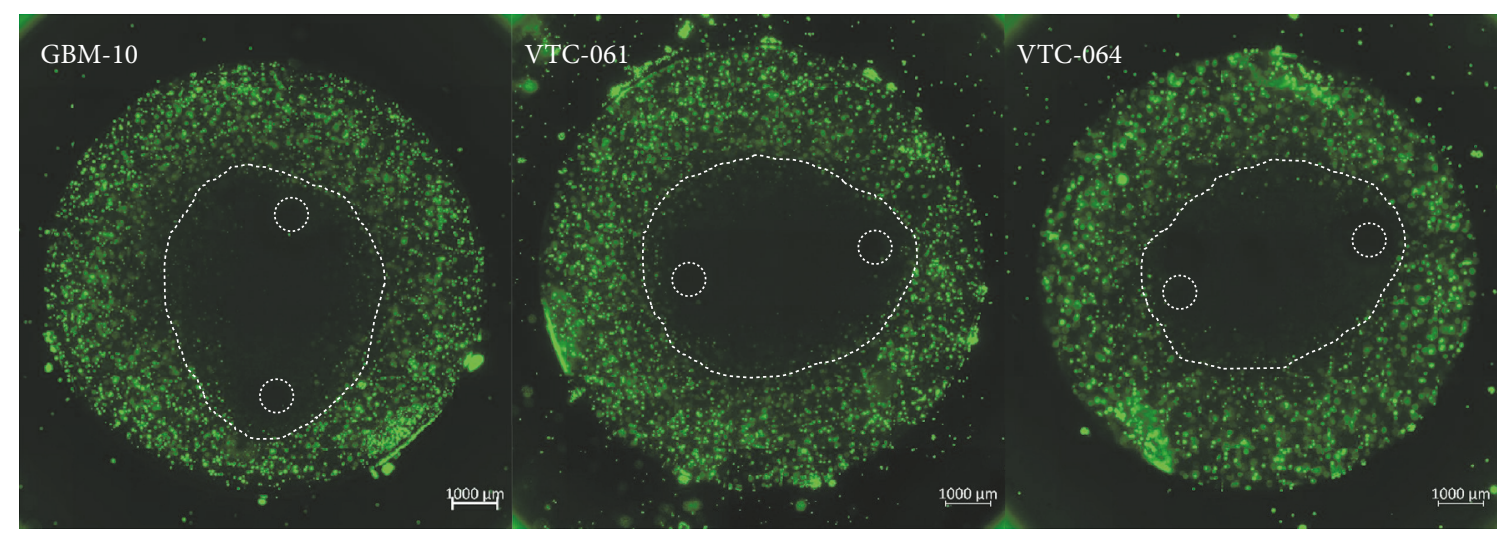

(a)

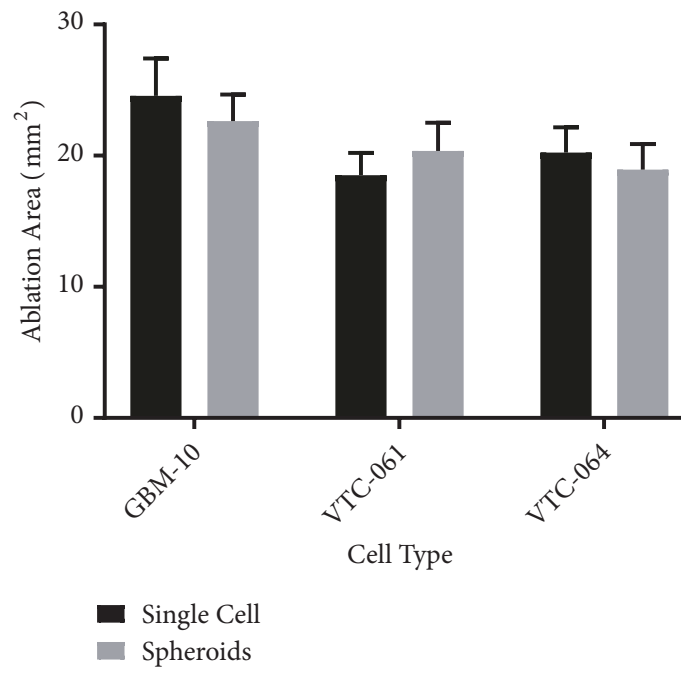

(b)

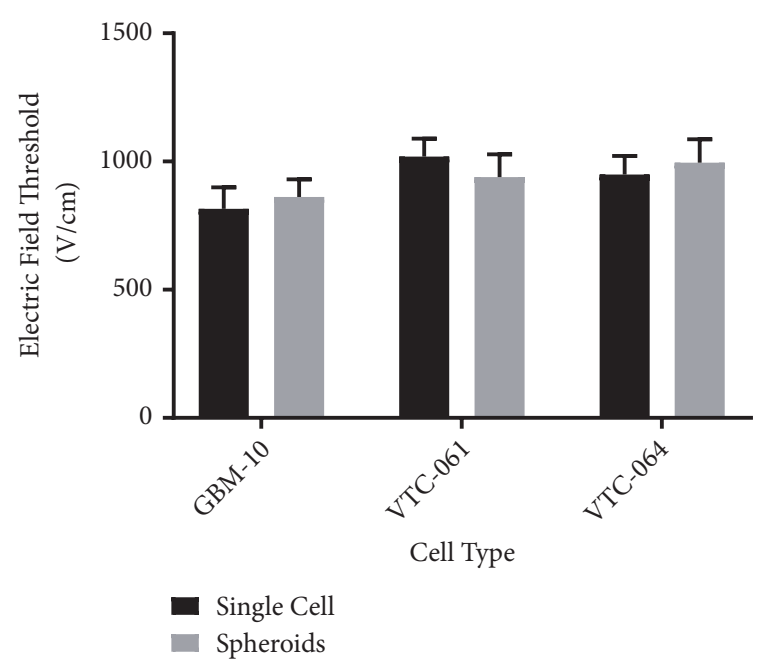

(c)

FIGURE 3: GSCs grown in multicellular spheroids in hydrogels exhibit similar H-FIRE response to single cells seeded in hydrogels. (a) H-FIRE lesions for GSCs grown into multicellular spheroids. (b) Comparison of single cell and spheroid hydrogels shows no significant difference in H-FIRE ablation areas. (c) Lethal electric field thresholds for treatment of GSCs do not change when treating spheroids versus single cells in collagen hydrogels.

been shown to better mimic in vivo biological and clinical behavior of GSCs in terms of both stem cell expression and drug response [56, 57]. Therefore, we chose HA as the biomaterial for these experiments. Cells seeded within the HA all exhibited a highly rounded morphology (Figure 4(a)). Because GBM-10 cells exhibited the lowest cell death electric field threshold in collagen among the patient-derived cells studied, these were selected for continued experiments in the HA scaffolds. In this context the GSC population, GBM 10, had similar overall cell areas to the bulk tumor cells, U-251, and the nonmalignant NHA cells when seeded within the HA (Figure 4(b)), thereby simplifying comparisons among these cell types. The nuclear areas of the malignant cells were all significantly greater than the nonmalignant NHAs. This results in NCR values for both GBM-10 and U-251 significantly greater than NHAs (Figure 4(c)). Unlike when seeded in collagen, the different cell types seeded in HA hydrogels all exhibit highly rounded morphology with similar circularities and aspect ratios (Figure 4(d)). Because cells seeded in HA all exhibit a morphology similar to the GSCs in collagen, while maintaining the trends of NCR and nuclear size, these hydrogels were used to test the robustness of the trends seen in collagen hydrogels treated with H-FIRE.

2.4. GSC Selectivity Trends Hold in HA Scaffolds. To test the effect of the altered ECM and resulting cell phenotype and morphology on electric field thresholds with H-FIRE therapy, cell-seeded HA scaffolds were exposed to electroporation protocols (Figure 5(a)). The ablation area achieved when GBM-10 scaffolds were exposed to H-FIRE was significantly greater than the ablation area of U-251 cells. Both GBM-10 cells and U-251 cells exhibited a significantly larger ablation area than NHA cells. The electric field thresholds derived from the ablation areas are $869 \pm 42 \mathrm{~V} / \mathrm{cm}$ for GBM-10 cells, $976 \pm 97 \mathrm{~V} / \mathrm{cm}$ for U-251 cells, and $1568 \pm 133 \mathrm{~V} / \mathrm{cm}$ for NHA cells. The selectivity trends seen in collagen remain for cells seeded in HA as GBM-10 lethal electric field thresholds are significantly lower than both U251 and NHA cells, while U-251 cells have a significantly lower lethal threshold than NHAs. 

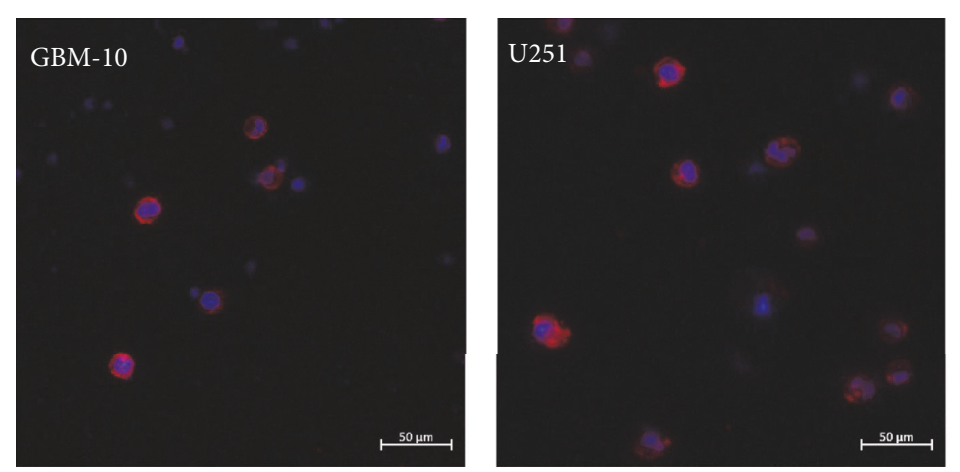

(a)

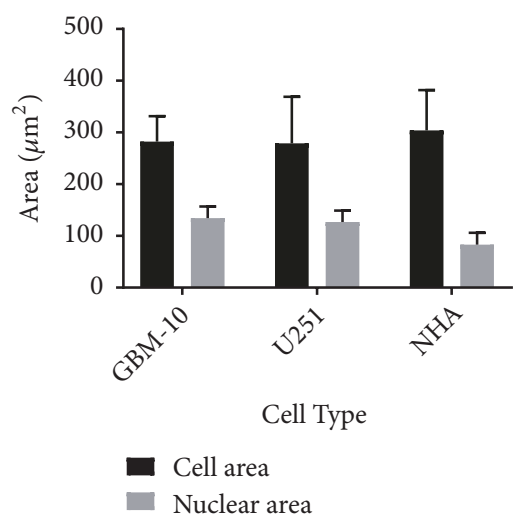

(b)

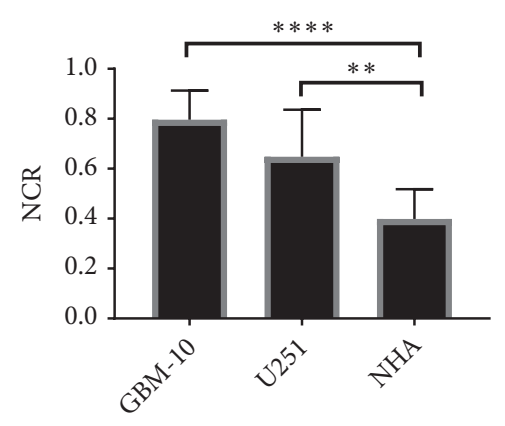

Cell Type (c)
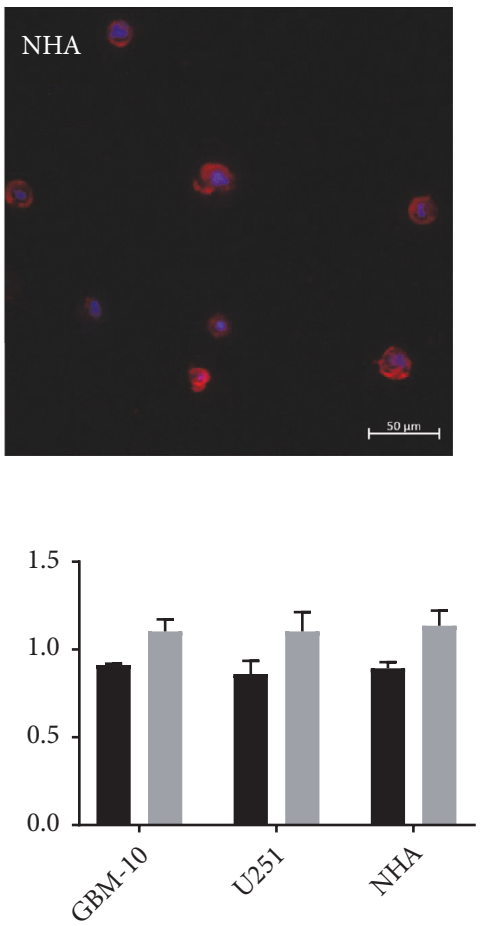

Cell Type

- Circularity Aspect Ratio

(d)

FIGURE 4: Cells seeded in hyaluronic acid hydrogels exhibit more uniform morphologies compared to collagen-seeded cells. (a) Confocal images of cells seeded in HA hydrogels show a rounded and compact cell morphology regardless of cell type. (b) Cells seeded in HA have similar cell areas ( $p>0.05)$ while malignant cells have larger nuclear areas than healthy cells $(p<0.01)$. (c) GSCs and U251 cells exhibit a larger NCR than NHAs: ${ }^{* *} \mathrm{p}<0.01 ;{ }^{* * *} \mathrm{p}<0.001$. (d) All three cell types exhibit similar circularity $(\mathrm{p}>0.05)$ and aspect ratios $(\mathrm{p}>0.05)$.

We next combined results from both the collagen and the HA scaffolds into a single graphical summary (Figure 6). Plotting lethal electric field threshold versus nuclear area shows a close correlation between the two variables with cells with larger nuclei requiring less energy to ablate, across all cell types (Figure 6(a)). This trend holds even when the scaffold material is changed and overall cell morphology is changed significantly as a result. The relationship between lethal electric field threshold and NCR has an overall similar trend with a decreasing electric field necessary for ablation as NCR increases. However, this dependence is not as strong as is observed for the dependence on nuclear area, and there were some cases where significant variation in NCR did not result in altered electric field threshold (Figure 6(b)).

\section{Discussion}

The results of our work determine that the GSC cells lines studied here are susceptible to H-FIRE ablation to a similar degree as differentiated tumor cells and are significantly more susceptible than normal astrocytes. Interestingly, two of the GSC populations tested (VTC-061 and VTC-064) have been shown previously to be resistant to chemotherapeutics [58]. Therefore, the ablation of these cell populations represents a potential means of targeting radiation and chemotherapy resistant cells that may repopulate tumors if found in vivo. However, based on our results, NSCs present in the immediate surroundings of the tumor space may be undesirably targeted by H-FIRE treatment. NSCs reside mostly in the hippocampus and the subventricular zone of the adult brain [59-61]. Hence, H-FIRE pulses must be applied with care in these areas. Our findings highlight the importance of considering the impact on tissue stem cell populations when utilizing H-FIRE procedures in other tissues.

In our previous studies $[58,62,63]$, we isolated and characterized glioblastoma stem cells from patient specimens. GSCs were cultured using sphere-forming assay. Next, the stem cell identity of these primary lines was verified using cell dilution assay and differentiation assay. By probing GBM stem-like cell lines with nestin (a well-established stem cell marker for GBM stem-like cells), we found that nestin was differentially expressed among glioblastoma stemlike cell lines and the levels of nestin did not correlate with the capability of GBM stem-like cells to self-renew. Our results strongly suggest that glioblastoma stem cells are heterogenous. Using sphere-forming assay for isolating GSCs does not guarantee the purity of GSC culture. However, given the lack of definitive markers for cancer stem cells from 


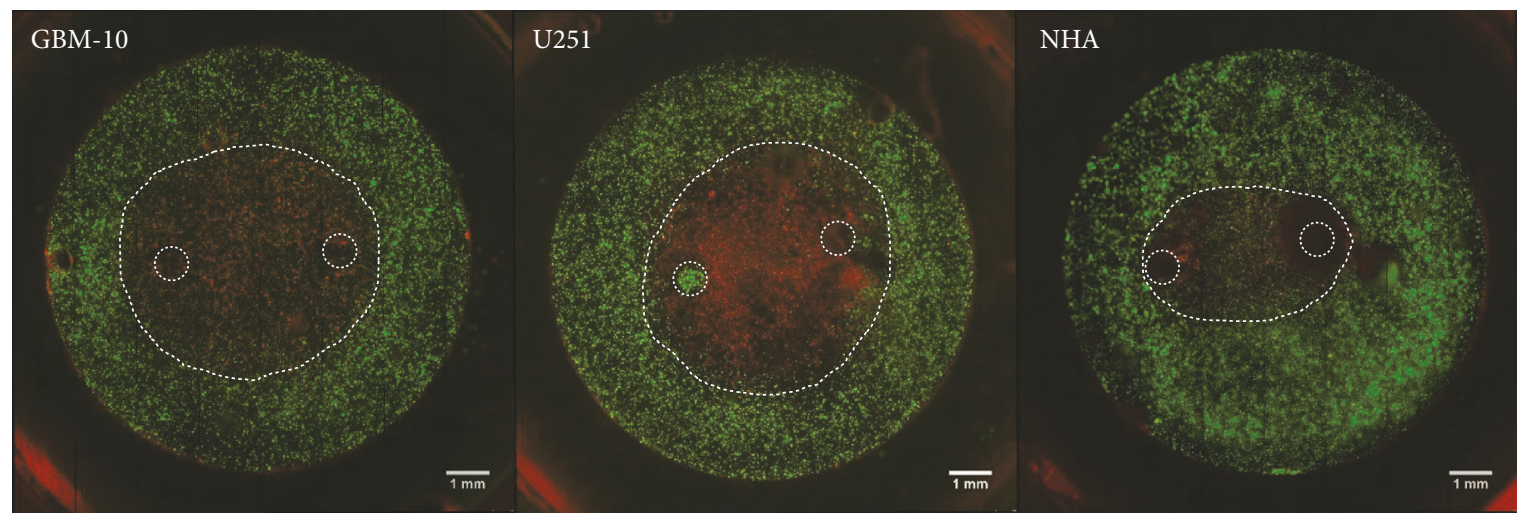

(a)

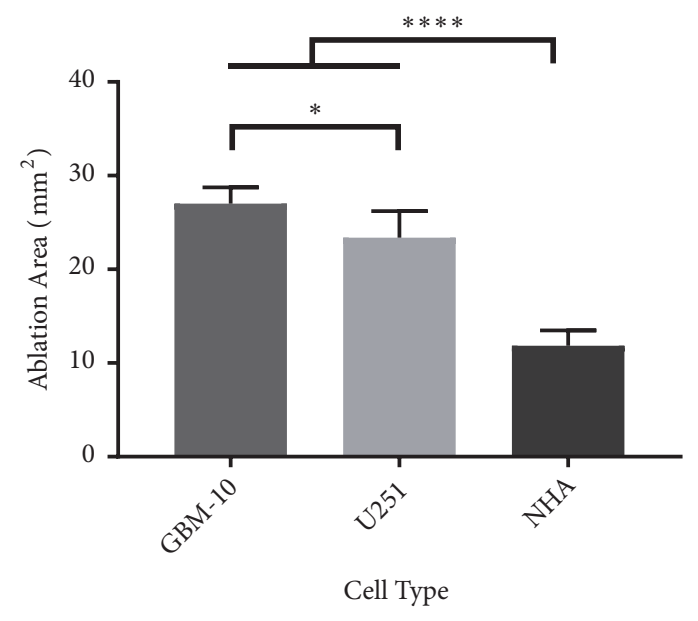

(b)

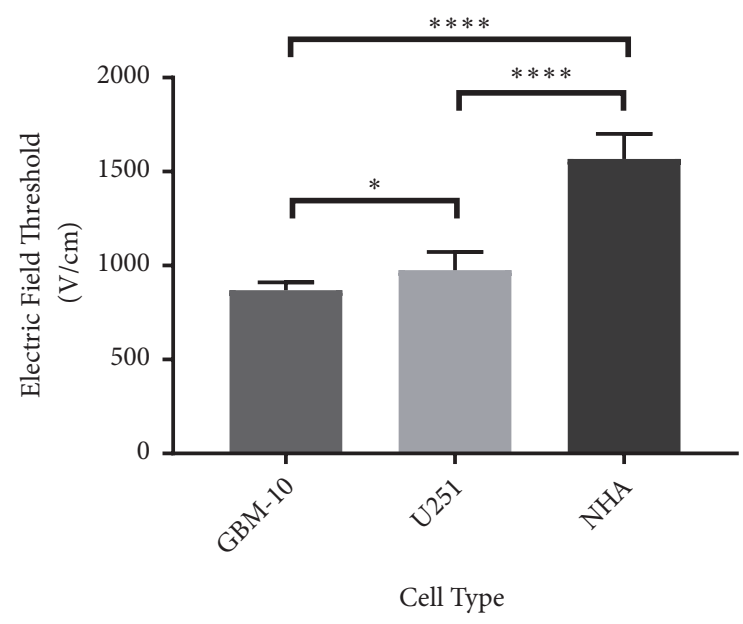

(c)

FIGURE 5: The ablation of GBM-10 GSCs is accomplished at lower thresholds than other cell types in hyaluronic acid. (a) Lesion areas after H-FIRE electroporation protocols in HA are visualized with live/dead staining. (b) Comparison of lesion areas in HA shows the GBM-10 GSC populations have a greater lesion size than U251s which has a greater lesion size than NHAs. (c) GSC populations in HA have a lower lethal threshold than healthy astrocytes and slightly lower but similar thresholds to U251 cells when exposed to H-FIRE pulses. ${ }^{*} \mathrm{p}<0.05$; **** $\mathrm{p}<0.0001$.

GBM, we argue that sphere-forming assay would be more reliable than marker-based cell sorting. For example, several prior reports show that CD133 negative glioblastoma cells (a classical cancer stem cell marker) also exhibit stem-like cell characteristics [64-66]. As such, sphere-forming assay retains heterogeneity within the populations of GBM stemlike cells, which may provide a better comparison between heterogenous GBM stem-like cells and astrocytes. In order to acquire unbiased results, we used heterogenous GBM stemlike cells in this study to compare the effect of H-FIRE between tumor and normal cells. Whether the properties of GSCs or their genetic heterogeneity results in the differences in H-FIRE treatment we observed that further investigation will be required.

We have previously suggested that both nuclear size as well as NCR may be important regulators of cell death in response to H-FIRE, as both of these parameters were previously shown to correlate with measured electric field cell death thresholds $[48,67]$. Interestingly, our comparison between collagen and HA scaffolds provided some test cases in which there was a large change in NCR yet with minimal change in lethal threshold observed. For example, U-251 cells cultured in collagen have an elongated morphology and therefore have a smaller NCR than when cultured in HA, in which the U-251s have a compact morphology. Yet U-251 cells have similar H-FIRE thresholds in both of these contexts, and in both instances exhibit a lower electric field threshold for ablation than NHAs. This result is seemingly difficult to reconcile with our previous results demonstrating enhanced $\mathrm{H}$-FIRE response with an ephrin signaling-induced increase in NCR for malignant cells [67]. While our current results confirm a general trend between electric field threshold and NCR, these results suggest a stronger relationship between electric field threshold and nuclear area. In the case of comparing thresholds in collagen and HA, cell-ECM interactions may furthermore play an important role, making comparison across scaffolding more challenging. Additionally, other signaling changes upon ephrin-activation may also be important in the context of IRE or H-FIRE induced cell death (e.g., altered cytoskeleton integrity [68]), such that the ephrin effect 


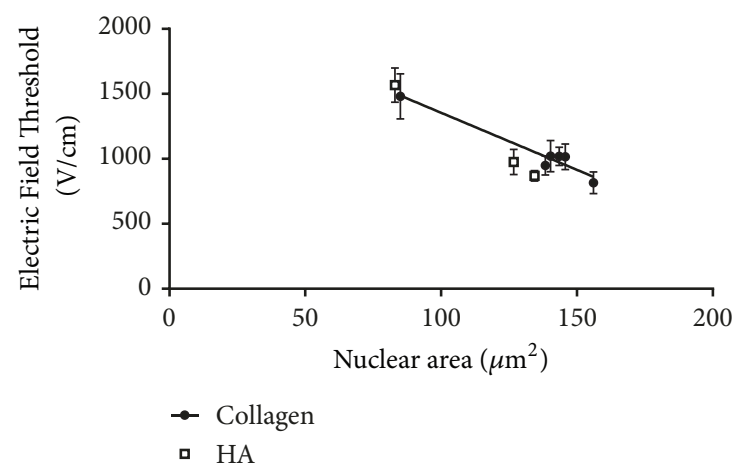

(a)

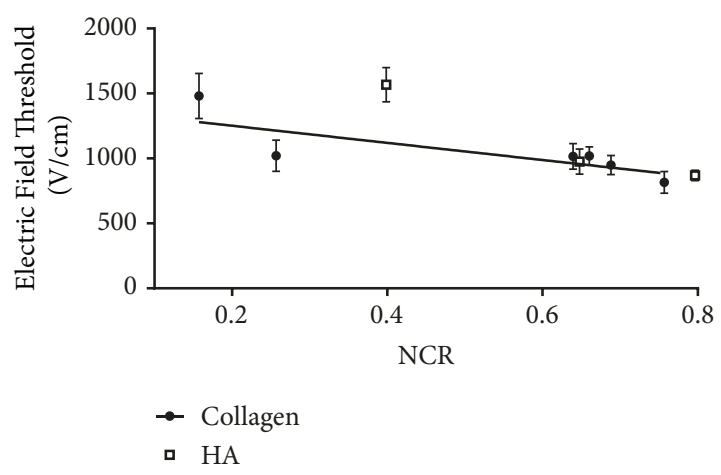

(b)

FIGURE 6: Summary of data in collagen and HA scaffolds. (a) A clear relationship emerges between nuclear area and lethal electric field threshold in both collagen and HA where cells with larger nuclei are ablated at lower electric field thresholds $\left(\mathrm{r}^{2}=0.89\right.$ linear correlation). (b) The relationship between NCR and electric field threshold is described by a reciprocal relationship, but with a significantly weaker dependence on NCR $\left(r^{2}=0.57\right.$ linear correlation).

on thresholds observed in previous work may not have been purely due to morphological changes. It should also be noted that, while cell size and morphology are crucial factors in determining the onset of electroporation, they are not the only important aspects for resulting cell death thresholds. Cells are permeabilized based on their morphology and 3D orientation; however, cell viability is also a function of their susceptibility to loss of homeostasis. These complex relationships between morphology and electric field threshold $[69,70]$ suggest that future work needs to include additional normal cell types with large nuclei as well as tumor cells with small nuclei, with the understanding that a range of cellular or tissue characteristics (e.g., metabolism, extracellular ionic concentrations [71, 72], and tissue conductivity) may be important additional factors in regulating cell death.

It is important to note that while the GSCs presented here exhibit the enlarged NCR seen in vivo [52], the morphology of these cells in the collagen hydrogel does not reflect the morphology of these cells in vivo. This is not especially surprising, as tumor stem cells tend to propagate as spheroids when cultured in vitro. Importantly for this study, the hydrogel platform was able to provide a $3 \mathrm{D}$ environment where cells experienced a range of electric fields, much like they would during an in vivo treatment. In addition, lethal thresholds can be easily determined using finite element modeling. However, collagen is not an ideal material for recapitulating stem cell properties found in vivo. Therefore, cells were also tested in hyaluronic acid hydrogels, a more physiologically relevant material $[56,57]$. Recreating the morphology of GSCs using in vitro models remains a challenge. Cells in our HA cultures retained spherical morphologies that are not representative of in vivo tissues but that are similar to other spheroid stem cell culture methods. Despite this lack of physiological relevance for GSC morphology in our hydrogel tissue mimics, the high NCR exhibited in our in vitro cultures of GSCs has been confirmed in vivo $[52,53,73]$. HA experiments furthermore provided a method to confirm that the lower electric fields necessary for ablation of GSCs in collagen gels were not due to a rounded morphology that may not be found in vivo. In HA scaffolds, GSCs, bulk tumor cells, and healthy astrocytes all take on a similar rounded morphology; however cell typespecific variations in cell death thresholds remained as in collagen. Therefore, we can conclude that the low electric field thresholds seen with GSC cells are not due to their circular morphology and may well be conserved if they were to take on a different morphology in vivo. However future in vivo experiments will be necessary to test this hypothesis.

The results of this study confirm and expand on our previous results demonstrating that a lower $\mathrm{H}$-FIRE threshold is required for ablation of malignant cells compared with important bulk cell types in the context of GBM. The results of this study add robustness to this conclusion as all previous malignant cells used were established and differentiated cell lines. This study demonstrates the feasibility of using patientderived cells for therapy testing to determine the different thresholds needed for various patient samples. The results of this study may be of high clinical relevance because they suggest an option for treatment of cells currently considered therapy-resistant. We have demonstrated successful and selective ablation of two populations of cells previously shown to be highly resistant to chemotherapy (VTC-064 and VTC061) $[23,58]$. These results suggest that H-FIRE may be a valuable therapy to be used in conjunction with more traditional therapies to reduce the population of resistant cells that may be left behind to cause tumor recurrence. Studies have shown cancer stem-like cells usually exist in a quiescent state but the population may grow exponentially when stimulated by surgery, chemotherapy, and radiotherapy [74]. Further studies will be done to ensure H-FIRE does not produce an increase in growth kinetics of these GSCs. If no such effect is found, it may be a valuable practice to follow surgery, chemotherapy, and radiotherapy used in GBM treatment with a regime of H-FIRE pulses. This may protect against GSCs causing a recurrent tumor as a result of therapy resistance and increased growth.

\section{Materials and Methods}

4.1. Cell Culture. GBM-10, VTC-061, and VTC-064 patientderived glioma stem cells were received from the lab of Dr. Zhi Sheng. These cells were isolated from resected tumor 


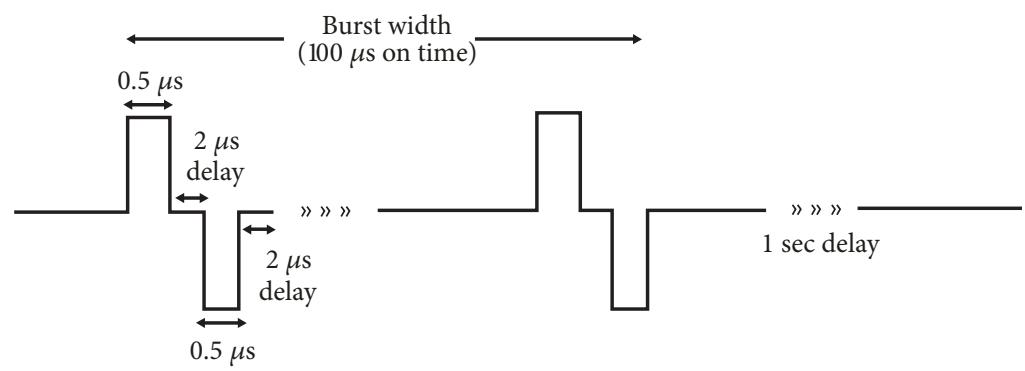

FIGURE 7: Schematic of H-FIRE waveforms used in the study. Collagen scaffolds were treated with a series of 50 bursts delivered at a frequency of one burst/second and amplitude of $800 \mathrm{~V}$ for a total on time of $100 \mu \mathrm{s}$. HA scaffolds were treated with the same parameters at an applied voltage of $950 \mathrm{~V}$.

tissue as described previously [62]. GBM-10, VTC-061, and VTC-064 cells were cultured as free-floating neurospheres in Dulbecco's Modified Eagle Medium (DMEM) (ATCC, Manassas, VA) supplemented with B27 (Life Technologies, Carlsbad, CA), $20 \mathrm{ng} / \mathrm{mL}$ epidermal growth factor (EGF) (Life Technologies, Carlsbad, CA), $20 \mathrm{ng} / \mathrm{mL}$ basic fibroblast growth factor (bFGF) (Life Technologies, Carlsbad, CA), 1\% L-glutamine (Life Technologies, Carlsbad, CA), and 1\% Penicillin/Streptomycin (PS) (Lonza, Basel, Switzerland). Normal Human Astrocyte (NHA) cells (Lonza, Basel, Switzerland) were cultured in Astrocyte Growth Media (Lonza, Basel, Switzerland). U-251 MG human glioblastoma cells (ATCC, Manassas, VA) were grown in DMEM containing 10\% FBS, $1 \%$ PS, and $0.1 \mathrm{mM}$ nonessential amino acid. Human Neural Stem cells (NSCs) were cultured as nonadherent neurospheres in StemPro ${ }^{\mathrm{TM}}$ NSC SFM (Thermo Fischer Scientific, Waltham, MA). All cells were grown in culture at $37^{\circ} \mathrm{C}$ in $5 \% \mathrm{CO}_{2}$ in a humidified incubator. Cells were seeded in hydrogels at a density of $1 \times 10^{6}$ cells $/ \mathrm{mL}$. The hydrogels were submerged in appropriate growth media for the cell type at $37^{\circ} \mathrm{C}$ in $5 \% \mathrm{CO}_{2}$ in a humidified incubator and cell viability was maintained within hydrogels for up to 14 days.

4.2. Construction of Collagen Scaffolds. Stocks of type I collagen were prepared by dissolving rat tail tendon in acetic acid, followed by freezing and lyophilization as described previously [75]. Stock solution concentrations of collagen were created at a density of $10 \mathrm{mg} / \mathrm{mL}$. Scaffolds with a final concentration of $5 \mathrm{mg} / \mathrm{mL}$ were made from concentrated collagen stocks to create collagen gels of $0.5 \%(\mathrm{w} / \mathrm{w})$. Neutralized collagen solutions were created by mixing acid-dissolved collagen with 10X DMEM (10\% of total collagen solution volume) and sufficient volumes of $1 \mathrm{~N} \mathrm{NaOH}$ until a pH in the range of 7.0-7.4 was achieved. The neutralized collagen was mixed with cells suspended in DMEM, NHA, NSC, or GSC media to achieve a cell density of $1 \times 10^{6}$ cells $/ \mathrm{mL}$ in the final collagen mixture. Solutions were mixed carefully with a sterilized spatula to ensure homogenous distribution throughout the gel without damaging cells. Collagen solutions were then dispensed into a polydimethylsiloxane (PDMS) mold with a cut-out of $10 \mathrm{~mm}$ diameter and $1 \mathrm{~mm}$ depth and molded flat to ensure consistent scaffold geometry. Our previous mathematical modeling and experiments on oxygen $\left(\mathrm{O}_{2}\right)$ consumption rates by tumor cells [76] confirm that, at this cell density and scaffold thickness, $\mathrm{O}_{2}$ concentration is uniform throughout the scaffold depth. Collagen was allowed to polymerize at $37^{\circ} \mathrm{C}$ and $5 \% \mathrm{CO}_{2}$ for 30 minutes. For testing of GSCs neurosphere morphology, hydrogels were seeded with single cells at a cell density of $1 \times 10^{5}$ cells $/ \mathrm{mL}$ and maintained in culture for 9 days to allow time for large neurospheres to grow from the individual cells.

4.3. Construction of Hyaluronic Acid Scaffolds. GBM-10, U251, and NHA cells were cultured in hyaluronic acid Hystem-C hydrogels (ESI Bio, Alameda, CA) according to the manufacturer's instructions. Prior to fabricating the 3D HA scaffolds, cells were removed from their flask using trypsin (Life Technologies, Carlsbad, CA) and centrifuged at $900 \mathrm{rpm}$ for $6 \mathrm{~min}$ to form a cell pellet. Cells pellets and resuspended in an equal parts mixture of glycosil (thiol-modified sodium hyaluronate) and Gelin-S (thio-modified gelatin). To form a hydrogel, the solution was mixed with Extralink (PEGDA, polyethylene glycol diacrylate) and allowed to crosslink in a $37^{\circ} \mathrm{C}$ incubator with $5 \%$ carbon dioxide for 30 minutes. After hydrogels were crosslinked, appropriate culture media for the seeded cell type were added to the well and gels were placed in the incubator and maintained until treatment. Cells were seeded in the Hystem-C hydrogel to create a final concentration of $1 \times 10^{6}$ cells $/ \mathrm{mL}$.

4.4. Fluorescent Staining. GBM-10, VTC-064, VTC-061, U251, NSC, and NHA cells were separately seeded in hydrogels described earlier. After culturing the cells for 24 hours, the hydrogels were fixed using $4 \%$ formalin and permeabilized and blocked using $40 \mathrm{mg} / \mathrm{mL}$ bovine serum albumin (BSA) and $0.05 \%$ Triton-X. Cellular F-actin was stained with Alexa Flour 568 phalloidin (Life Technologies, Carlsbad, CA) while cell nuclei were stained with diaminophenylindole (DAPI; Sigma-Aldrich, St. Louis, MO). Cells were visualized using a Zeiss LSM880 (Carl Zeiss Microscopy LLC, Thornwood, NY) laser scanning confocal microscope.

4.5. Determination of NCR. Fluorescent stained cells were used to determine overall cell area and nuclear area for cells. Image analysis was done in Image J (NIH, Bethesda, MD). Z-stack images were converted into $2 \mathrm{D}$ projection images 
and cell measurements were made from these projections. Measurements were made on at least four cells from different area of the hydrogel (20 cells total) and at least 5 hydrogels were analyzed for each condition. NCR was calculated from the measured cell area $\left(A_{C}\right)$ and nuclear area $\left(A_{N}\right)$ as follows:

$$
N C R=\frac{A_{N}}{A_{C}-A_{N}}
$$

Aspect ratio was calculated from the measured largest diameter (dmax) and smallest diameter orthogonal to dmax (dmin) as follows:

$$
A_{R}=\frac{d_{\text {min }}}{d_{\text {max }}}
$$

Circularity of the cells was calculated from the measured perimeter of the cell $(\mathrm{P})$ and overall cell area $\left(\mathrm{A}_{\mathrm{C}}\right)$ as follows:

$$
f_{\text {circ }}=\frac{4 \pi A_{C}}{P^{2}}
$$

The scaffolds that all cells were seeded in are isotropic and therefore there was no observed orientation dependence on this $2 \mathrm{D}$ projection from the $3 \mathrm{D}$ morphology.

4.6. Electroporation of 3D Scaffolds. Pulsed electroporation experiments were performed in hydrogels with constant electrical properties. The electrical conductivities of each of the collagen gel-cell mixtures were measured before crosslinking, using a conductivity meter (B-173, Horiba, Kyoto, Japan) to ensure similar electrical properties $(0.98 \pm 0.04 \mathrm{~S} / \mathrm{m})$. H-FIRE pulses were delivered using a custom-built pulse generation system (INSPIRE 2.0, VoltMed Inc., Blacksburg, VA). Media were aspirated from hydrogels prior to exposure to treatment. Two solid stainless-steel cylinders with diameters of $0.87 \mathrm{~mm}$, separated $3.3 \mathrm{~mm}$ edge-to-edge, were used as electrodes. These electrodes were inserted through the full thickness of the hydrogel. These electrodes were connected to the pulse generation system, which was programmed to deliver treatment according to the H-FIRE protocol. For the H-FIRE protocol used, bursts consisting of $200 \times 500 \mathrm{~ns}$ pulses with a $2 \mu$ s interpulse delay were delivered with a repetition rate of 1 burst per second for a total of 50 bursts. For cells seeded in collagen, a peak voltage of $800 \mathrm{~V}$ was used to produce ablations in the hydrogel large enough for distinct electric field lines to be measured but small enough that boundary effects of the hydrogel edge did not interfere with the electric field distribution at the lethal threshold. In treating cells seeded in HA hydrogels, a peak voltage of $950 \mathrm{~V}$ was used.

4.7. Finite Element Analysis in Hydrogels. Finite element models using COMSOL Multiphysics (Version 4.3, COMSOL Inc., Palo Alto, CA) were used to solve the Laplace equation to find the electric field distribution within the hydrogels for each different voltage used. COMSOL Multiphysics was also used to solve the Joule heating equation to calculate the temperature distribution in the hydrogel as a result of each treatment. The simulation geometry was modeled as a $10 \mathrm{~mm}$ diameter and $1 \mathrm{~mm}$ thick cylinder with two steel electrode cylinders $(\mathrm{d}=0.87 \mathrm{~mm})$ spanning the depth of the hydrogel (Figure 8(a)). Thermal and electrical properties for each domain have been described previously [77]. The mesh was refined until error between successive refinements was less than $1 \%$. The final mesh contained 47,438 elements and solutions were found in approximately 3 minutes on a Pentium i3 processor.

4.8. Determination of Lethal Threshold. The thresholds for cell death were determined by analyzing images taken of livedead stain on the hydrogels 24 hours after delivering treatment. Hydrogel scaffolds were incubated in $2 \mu \mathrm{M}$ Calcein AM (Biotium, Hayward, CA) and $4 \mu \mathrm{M}$ ethidium homodimer III (Biotium, Hayward, CA) in PBS for 30 minutes at room temperature. Live cells were labeled green with calcein AM staining while cells lacking membrane integrity were labeled red with ethidium homodimer III and were considered dead. Images of each hydrogel were captured used a Zeiss LSM 880 microscope using a $5 \mathrm{X}$ objective and pieced together by automatic stitching. A custom algorithm developed in MATLAB was used to measure the lesion area from the imported images. Lesion areas were determined by analyzing intensity of points from the green channel in MATLAB as described previously [78]. A threshold of $20 \%$ of the maximum intensity value was used to separate the live region from the dead region in each image. Geometric measurements of the ablation zones were mapped to a finite element model developed in COMSOL Multiphysics 5.3 (Stockholm, Sweden) as described previously [48] (Figure 8(b)). Numerically integrating the surface of the scaffold across a range of electric field magnitudes in the finite element model allows for correlating ablation area to lethal electric field threshold (Figure 8(c)). A sixth-order polynomial found with least squares fitting in MATLAB resulted in a maximum relative error of $4.7 \%$ between electric field from the numerical fit and the COMSOL model. Use of this polynomial fit allows for a calculation of the electric field threshold for each ablation area from the COMSOL model for the scaffold. The lethal electric field threshold reported is the electric field distribution at the edge of the measured lesion area for the designated electrode and hydrogel configuration. It should be noted that the thresholds calculated here show the minimum field needed to cause cell death. The minimum field needed to induce electroporation is not calculated here.

4.9. Statistical Analysis. Statistical significance was determined by a two-tailed $t$-test performed in Prism Statistical Software (Version 6, Graphpad, La Jolla, CA). A 95\% confidence interval was used with significance defined as $\mathrm{p}<$ 0.05. All numerical results are reported as the mean and the standard deviation of all experimental measurements. No outliers were excluded.

\section{Conflicts of Interest}

J. W. Ivey, R. V. Davalos, and S. S. Verbridge are inventors on pending and issued patents related to the work. The authors declare no other competing financial interest. 


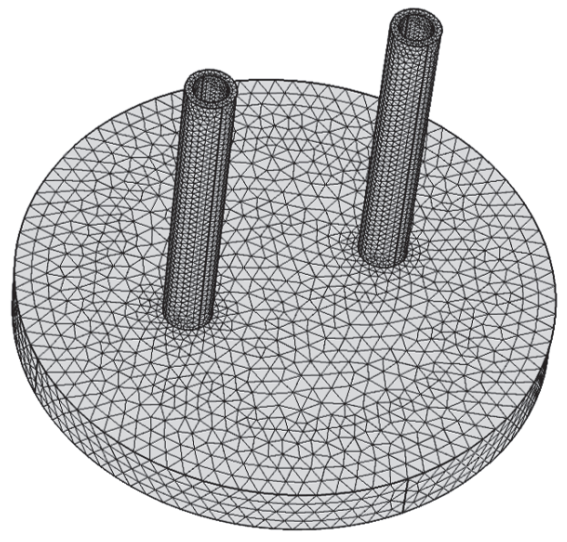

(a)

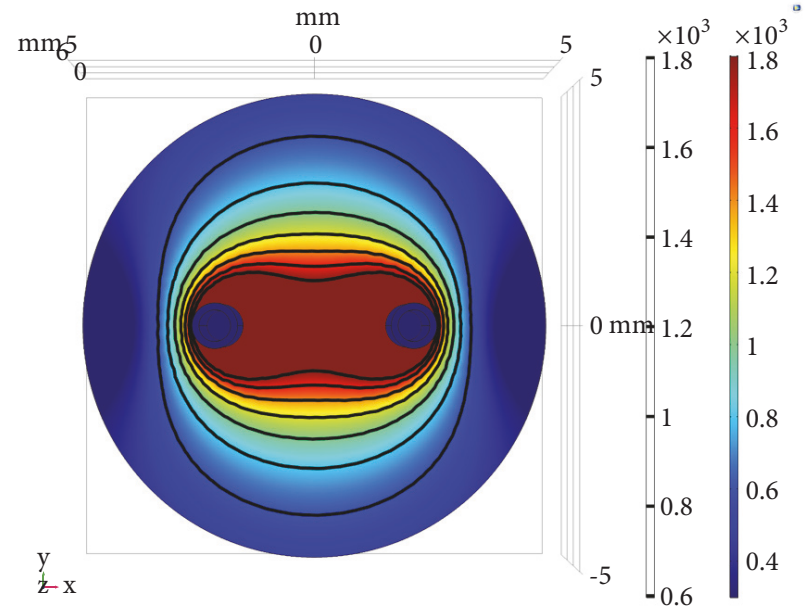

(b)

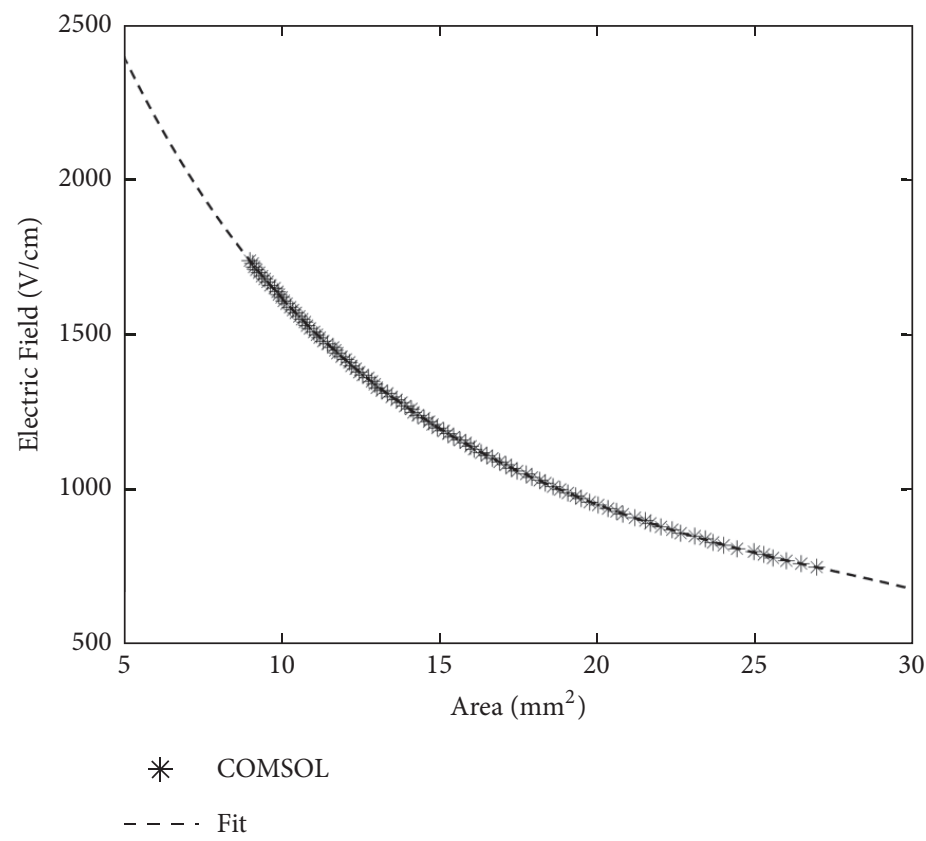

(c)

FiguRE 8: Finite element model relates ablation area to electric field threshold in hydrogel system. (a) Geometry and meshing of the finite element model used to simulate treatment of the hydrogel platform. (b) COMSOL model of hydrogel exposed to H-FIRE treatment (800V) shows how electric field changes across the scaffold. (c) Numerical integration of the finite element model of the electric field distribution in the scaffold allows for correlating ablation area to lethal electric field threshold.

\section{Authors' Contributions}

J. W. Ivey was responsible for study design, cell culture, 3D scaffold construction, confocal microscopy imaging, livedead staining, mathematical modeling, data analysis and interpretation, and writing of manuscript. E. M. Wasson was responsible for custom algorithm for area quantification, data analysis, and writing of manuscript. A. Kanitkar was responsible for cell culture, 3D scaffold construction, confocal microscopy imaging, live-dead staining, and data analysis and interpretation. W. Debinski was responsible for data analysis and interpretation of manuscript. N. Alinezhadbalalami was responsible for data analysis and interpretation and writing of manuscript. Z. Sheng was responsible for data analysis and interpretation and writing of manuscript. R. V. Davalos was responsible for data analysis and interpretation and writing of manuscript. S. S. Verbridge was responsible for conception of project plan, study design, data analysis and interpretation, and writing of manuscript.

\section{Acknowledgments}

This work was supported by the National Cancer Institute of the National Institutes of Health through awards R21CA192042, R01CA213423, and P01CA207206 and by 
a National Science Foundation CAREER Award (CBET1652112).

\section{References}

[1] S. A. Grossman, X. Ye, S. Piantadosi et al., "Survival of patients with newly diagnosed glioblastoma treated with radiation and temozolomide in research studies in the United States," Clinical Cancer Research, vol. 16, no. 8, pp. 2443-2449, 2010.

[2] R. Stupp, W. P. Mason, M. J. van den Bent et al., "Radiotherapy plus concomitant and adjuvant temozolomide for glioblastoma," The New England Journal of Medicine, vol. 352, no. 10, pp. 987-996, 2005.

[3] J. Clarke, N. Butowski, and S. Chang, "Recent advances in therapy for glioblastoma," Archives of Neurology, vol. 67, no. 3, pp. 279-283, 2010.

[4] R. Stupp, J.-C. Tonn, M. Brada, G. Pentheroudakis, and the ESMO Guidelines Working Group, "High-grade malignant glioma: ESMO clinical practice guidelines for diagnosis, treatment and follow-up," Annals of Oncology, vol. 21, no. 5, pp. v190v193, 2010.

[5] R. Stupp, M. E. Hegi, W. P. Mason et al., "Effects of radiotherapy with concomitant and adjuvant temozolomide versus radiotherapy alone on survival in glioblastoma in a randomised phase III study: 5-year analysis of the EORTC-NCIC trial," The Lancet Oncology, vol. 10, no. 5, pp. 459-466, 2009.

[6] P. Y. Wen and S. Kesari, "Malignant gliomas in adults," The New England Journal of Medicine, vol. 359, pp. 492-507, 2008.

[7] R. Bonavia, M.-D. Inda, W. K. Cavenee, and F. B. Furnari, "Heterogeneity maintenance in glioblastoma: a social network," Cancer Research, vol. 71, no. 12, pp. 4055-4060, 2011.

[8] A. Sottoriva, I. Spiteri, S. G. M. Piccirillo et al., "Intratumor heterogeneity in human glioblastoma reflects cancer evolutionary dynamics," Proceedings of the National Acadamy of Sciences of the United States of America, vol. 110, no. 10, pp. 4009-4014, 2013.

[9] A. P. Patel, I. Tirosh, J. J. Trombetta et al., "Single-cell RNA-seq highlights intratumoral heterogeneity in primary glioblastoma," Science, vol. 344, no. 6190, pp. 1396-1401, 2014.

[10] M. Snuderl, L. Fazlollahi, L. P. Le et al., "Mosaic amplification of multiple receptor tyrosine kinase genes in glioblastoma," Cancer Cell, vol. 20, no. 6, pp. 810-817, 2011.

[11] N. J. Szerlip, A. Pedraza, D. Chakravarty et al., "Intratumoral heterogeneity of receptor tyrosine kinases EGFR and PDGFRA amplification in glioblastoma defines subpopulations with distinct growth factor response," Proceedings of the National Acadamy of Sciences of the United States of America, vol. 109, no. 8, pp. 3041-3046, 2012.

[12] M. Meyer, J. Reimand, X. Lan et al., "Single cell-derived clonal analysis of human glioblastoma links functional and genomic heterogeneity," Proceedings of the National Acadamy of Sciences of the United States of America, vol. 112, no. 3, pp. 851-856, 2015.

[13] A. Sottoriva, L. Vermeulen, and S. Tavare, "Modeling evolutionary dynamics of epigenetic mutations in hierarchically organized tumors," PLoS Computational Biology, vol. 7, no. 5, Article ID e1001132, 2011.

[14] A. Marusyk and K. Polyak, "Tumor heterogeneity: causes and consequences," Biochimica et Biophysica Acta (BBA) - Reviews on Cancer, vol. 1805, no. 1, pp. 105-117, 2010.

[15] C. T. Jordan, M. L. Guzman, and M. Noble, "Cancer stem cells," The New England Journal of Medicine, vol. 355, pp. 1253-1261, 2006.
[16] X. Fan, L. G. Salford, and B. Widegren, "Glioma stem cells: evidence and limitation," Seminars in Cancer Biology, vol. 17, no. 3, pp. 214-218, 2007.

[17] J. Ji, K. L. Black, and J. S. Yu, "Glioma stem cell research for the development of immunotherapy," Neurosurgery Clinics of North America, vol. 21, no. 1, pp. 159-166, 2010.

[18] G. Liu, X. Yuan, Z. Zeng et al., "Analysis of gene expression and chemoresistance of CD133+ cancer stem cells in glioblastoma," Molecular Cancer, vol. 5, p. 67, 2006.

[19] T. C. Johannessen, R. Bjerkvig, and B. B. Tysnes, "DNA repair and cancer stem-like cells-potential partners in glioma drug resistance?" Cancer Treatment Reviews, vol. 34, no. 6, pp. 558$567,2008$.

[20] E. Nakai, K. Park, T. Yawata et al., "Enhanced mdr1 expression and chemoresistance of cancer stem cells derived from glioblastoma," Cancer Investigation, vol. 27, no. 9, pp. 901-908, 2009.

[21] S. Bao, Q. Wu, R. E. McLendon et al., "Glioma stem cells promote radioresistance by preferential activation of the DNA damage response," Nature, vol. 444, no. 7120, pp. 756-760, 2006.

[22] J. Wang, T. P. Wakeman, J. D. Lathia et al., "Notch promotes radioresistance of glioma stem cells," Stem Cells, vol. 28, no. 1, pp. 17-28, 2010.

[23] K. P. Bhat, V. Balasubramaniyan, B. Vaillant et al., "Mesenchymal differentiation mediated by NF- $\kappa \mathrm{B}$ promotes radiation resistance in glioblastoma," Cancer Cell, vol. 24, no. 3, pp. 331346, 2013.

[24] S.-H. Kim, K. Joshi, R. Ezhilarasan et al., "EZH2 protects glioma stem cells from radiation-induced cell death in a MELK/FOXM1-dependent manner," Stem Cell Reports, vol. 4, no. 2, pp. 226-238, 2015.

[25] M. Venere, P. Hamerlik, Q. Wu et al., “Therapeutic targeting of constitutive PARP activation compromises stem cell phenotype and survival of glioblastoma-initiating cells," Cell Death of Differentiation, vol. 21, no. 2, pp. 258-269, 2014.

[26] J. M. Heddleston, Z. Li, R. E. McLendon, A. B. Hjelmeland, and J. N. Rich, "The hypoxic microenvironment maintains glioblastoma stem cells and promotes reprogramming towards a cancer stem cell phenotype," Cell Cycle, vol. 8, no. 20, pp. 32743284, 2009.

[27] Z. Li, S. Bao, Q. Wu et al., "Hypoxia-inducible factors regulate tumorigenic capacity of glioma stem cells," Cancer Cell, vol. 15, no. 6, pp. 501-513, 2009.

[28] A. B. Hjelmeland, Q. Wu, J. M. Heddleston et al., "Acidic stress promotes a glioma stem cell phenotype," Cell Death \& Differentiation, vol. 18, pp. 829-840, 2011.

[29] W. A. Flavahan, Q. Wu, M. Hitomi et al., "Brain tumor initiating cells adapt to restricted nutrition through preferential glucose uptake," Nature Neuroscience, vol. 16, no. 10, pp. 1373-1382, 2013.

[30] M. D. Sørensen, S. Fosmark, S. Hellwege, D. Beier, B. W. Kristensen, and C. P. Beier, "Chemoresistance and chemotherapy targeting stem-like cells in malignant glioma," in Stem Cell Biology in Neoplasms of the Central Nervous System, pp. 111-138, Springer, 2015.

[31] B. E. Stopschinski, C. P. Beier, and D. Beier, "Glioblastoma cancer stem cells - from concept to clinical application," Cancer Letters, vol. 338, no. 1, pp. 32-40, 2013.

[32] B. Yang, Y. Wang, C. Yang et al., "The ultrastructural difference between CD133-positive U251 glioma stem cells and normal U251 glioma cells," Ultrastructural Pathology, vol. 36, no. 6, pp. 404-408, 2012. 
[33] A. L. Vescovi, R. Galli, and B. A. Reynolds, "Brain tumour stem cells," Nature Reviews Cancer, vol. 6, pp. 425-436, 2006.

[34] A. Flemming, "Cancer stem cells: targeting the root of cancer relapse," Nature Reviews Drug Discovery, vol. 14, no. 3, p. 165, 2015.

[35] M. S. Wicha, “Targeting self-renewal, an Achilles' heel of cancer stem cells," Nature Medicine, vol. 20, no. 1, pp. 14-15, 2014.

[36] J. E. Visvader, “Cells of origin in cancer," Nature, vol. 469, no. 7330, pp. 314-322, 2011.

[37] B. Auffinger, A. L. Tobias, Y. Han et al., "Conversion of differentiated cancer cells into cancer stem-like cells in a glioblastoma model after primary chemotherapy," Cell Death \& Differentiation, vol. 21, pp. 1119-1131, 2014.

[38] R. C. Martin, D. Kwon, S. Chalikonda et al., “Treatment of 200 locally advanced (stage III) pancreatic adenocarcinoma patients with irreversible electroporation: safety and efficacy," Annals of Surgery, vol. 262, no. 3, pp. 486-494, 2015.

[39] J. H. Rossmeisl Jr., P. A. Garcia, T. E. Pancotto et al., "Safety and feasibility of the NanoKnife system for irreversible electroporation ablative treatment of canine spontaneous intracranial gliomas," Journal of Neurosurgery, vol. 123, no. 4, pp. 1008-1025, 2015.

[40] R. V. Davalos, B. Rubinsky, and L. M. Mir, "Theoretical analysis of the thermal effects during in vivo tissue electroporation," Bioelectrochemistry, vol. 61, no. 1-2, pp. 99-107, 2003.

[41] B. Al-Sakere, F. André, C. Bernat et al., “Tumor ablation with irreversible electroporation," PLoS ONE, vol. 2, no. 11, Article ID e1135, 2007.

[42] R. C. Lee, D. J. Canaday, and S. M. Hammer, "Transient and stable ionic permeabilization of isolated skeletal muscle cells after electrical shock," Journal of Burn Care \& Rehabilitation, vol. 14, no. 5, pp. 528-540, 1993.

[43] J. C. Weaver, "Electroporation: a general phenomenon for manipulating cells and tissues," Journal of Cellular Biochemistry, vol. 51, no. 4, pp. 426-435, 1993.

[44] M. L. Yarmush, A. Golberg, G. Sersa, T. Kotnik, and D. Miklavcic, "Electroporation-based technologies for medicine: principles, applications, and challenges," Annual Review of Biomedical Engineering, vol. 16, pp. 295-320, 2014.

[45] T. Kotnik, L. Rems, M. Tarek, and D. Miklavcic, "Membrane electroporation and electropermeabilization: mechanisms and models," Annual Review of Biophysics, vol. 48, 2019.

[46] C. B. Arena, M. B. Sano, J. H. Rossmeisl Jr. et al., "Highfrequency irreversible electroporation (H-FIRE) for nonthermal ablation without muscle contraction," Biomedical Engineering Online, vol. 10, article no. 102, 2011.

[47] S. P. Bhonsle, C. B. Arena, D. C. Sweeney, and R. V. Davalos, "Mitigation of impedance changes due to electroporation therapy using bursts of high-frequency bipolar pulses," Biomedical Engineering Online, vol. 14, supplement 3, 2015.

[48] J. W. Ivey, E. L. Latouche, M. B. Sano, J. H. Rossmeisl, R. V. Davalos, and S. S. Verbridge, "Targeted cellular ablation based on the morphology of malignant cells," Scientific Reports, vol. 5, no. 1, Article ID 17157, 2015.

[49] M. B. Sano, C. B. Arena, M. R. DeWitt, D. Saur, and R. V. Davalos, "In-vitro bipolar nano- and microsecond electro-pulse bursts for irreversible electroporation therapies," Bioelectrochemistry, vol. 100, pp. 69-79, 2014.

[50] P. A. Garcia, J. H. Rossmeisl, J. L. Robertson et al., "7.0-T magnetic resonance imaging characterization of acute bloodbrain-barrier disruption achieved with intracranial irreversible electroporation," PLoS ONE, vol. 7, no. 11, Article ID e50482, 2012.

[51] C. B. Arena, P. A. Garcia, M. B. Sano et al., "Focal blood-brainbarrier disruption with high-frequency pulsed electric fields," Technology, vol. 2, no. 3, pp. 206-213, 2014.

[52] Y. Zhao, Q. Huang, T. Zhang et al., "Ultrastructural studies of glioma stem cells/progenitor cells," Ultrastructural Pathology, vol. 32, no. 6, pp. 241-245, 2008.

[53] S. Yamamuro, Y. Okamoto, E. Sano et al., "Characterization of glioma stem-like cells from human glioblastomas," International Journal of Oncology, vol. 47, no. 1, pp. 91-96, 2015.

[54] J. Zamecnik, "The extracellular space and matrix of gliomas," Acta Neuropathologica, vol. 110, no. 5, pp. 435-442, 2005.

[55] L. W. Lau, R. Cua, M. B. Keough, S. Haylock-Jacobs, and V. W. Yong, "Pathophysiology of the brain extracellular matrix: a new target for remyelination," Nature Reviews Neuroscience, vol. 14, no. 10, pp. 722-729, 2013.

[56] L. Jadin, S. Pastorino, R. Symons et al., "Hyaluronan expression in primary and secondary brain tumors," Annals of Translational Medicine, vol. 3, no. 6, p. 80, 2015.

[57] S. J. Florczyk, K. Wang, S. Jana et al., "Porous chitosanhyaluronic acid scaffolds as a mimic of glioblastoma microenvironment ECM," Biomaterials, vol. 34, no. 38, pp. 10143-10150, 2013.

[58] P. Kanabur, S. Guo, G. R. Simonds et al., "Patient-derived glioblastoma stem cells respond differentially to targeted therapies," Oncotarget , vol. 7, no. 52, pp. 86406-86419, 2016.

[59] D. C. Lie, H. Song, S. A. Colamarino, G. L. Ming, and F. H. Gage, "Neurogenesis in the adult brain: new strategies for central nervous system diseases," Annual Review of Pharmacology and Toxicology, vol. 44, pp. 399-421, 2004.

[60] G. L. Ming and H. Song, "Adult neurogenesis in the mammalian central nervous system," Annual Review of Neuroscience, vol. 28, pp. 223-250, 2005.

[61] L. N. Manganas, X. Zhang, Y. Li et al., "Magnetic resonance spectroscopy identifies neural progenitor cells in the live human brain," Science, vol. 318, no. 5852, pp. 980-985, 2007.

[62] S. F. Murphy, R. T. Varghese, S. Lamouille et al., "Connexin 43 inhibition sensitizes chemoresistant glioblastoma cells to temozolomide," Cancer Research, vol. 76, no. 1, pp. 139-149, 2016.

[63] K. J. Pridham, L. Le, S. Guo et al., "PIK3CB/p110 $\beta$ is a selective survival factor for glioblastoma," Neuro-Oncology, vol. 20, no. 4, pp. 494-505, 2018.

[64] D. Beier, P. Hau, M. Proescholdt et al., "CD133' and CD133glioblastoma-derived cancer stem cells show differential growth characteristics and molecular profiles," Cancer Research, vol. 67, no. 9, pp. 4010-4015, 2007.

[65] C. P. Beier and D. Beier, "CD133 negative cancer stem cells in glioblastoma," Frontiers in Bioscience - Elite, vol. 3, no. 2, pp. 701710, 2011.

[66] C. Lottaz, D. Beier, K. Meyer et al., "Transcriptional profiles of $\mathrm{CD}_{133^{+}}$and $\mathrm{CD} 133^{-}$glioblastoma-derived cancer stem cell lines suggest different cells of origin," Cancer Research, vol. 70, no. 5, pp. 2030-2040, 2010.

[67] J. W. Ivey, E. L. Latouche, M. L. Richards et al., "Enhancing irreversible electroporation by manipulating cellular biophysics with a molecular adjuvant," Biophysical Journal, vol. 113, no. 2, pp. 472-480, 2017.

[68] I. Goswami, J. B. Perry, M. E. Allen, D. A. Brown, M. R. von Spakovsky, and S. S. Verbridge, "Influence of pulsed electric fields and mitochondria-cytoskeleton interactions on 
cell respiration," Biophysical Journal, vol. 114, no. 12, pp. 29512964, 2018.

[69] T. Murovec, D. C. Sweeney, E. Latouche, R. V. Davalos, and C. Brosseau, "Modeling of transmembrane potential in realistic multicellular structures before electroporation," Biophysical Journal, vol. 111, no. 10, pp. 2286-2295, 2016.

[70] G. Pucihar, T. Kotnik, B. Valic, and D. Miklavcic, "Numerical determination of transmembrane voltage induced on irregularly shaped cells," Annals of Biomedical Engineering, vol. 34, pp. 642-652, 2006.

[71] G. Pucihar, T. Kotnik, M. Kandušer, and D. Miklavčič, "The influence of medium conductivity on electropermeabilization and survival of cells in vitro," Bioelectrochemistry, vol. 54, no. 2, pp. 107-115, 2001.

[72] J. Dermol, O. N. Pakhomova, A. G. Pakhomov, and D. Miklavcic, "Cell electrosensitization exists only in certain electroporation buffers," PLoS One, vol. 11, no. 7, Article ID e0159434, 2016.

[73] J. Wang, H. Wang, Z. Li et al., "c-Myc is required for maintenance of glioma cancer stem cells," PLoS ONE, vol. 3, no. 11, Article ID e3769, 2008.

[74] I. A. Lubensky, A. O. Vortmeyer, S. Kim et al., "Identification of tumor precursor cells in the brains of primates with radiationinduced de novo glioblastoma multiforme," Cell Cycle, vol. 5, no. 4, pp. 452-456, 2006.

[75] V. L. Cross, Y. Zheng, N. Won Choi et al., "Dense type I collagen matrices that support cellular remodeling and microfabrication for studies of tumor angiogenesis and vasculogenesis in vitro," Biomaterials, vol. 31, no. 33, pp. 8596-8607, 2010.

[76] S. S. Verbridge, N. W. Choi, Y. Zheng, D. J. Brooks, A. D. Stroock, and C. Fischbach, "Oxygen-controlled three-dimensional cultures to analyze tumor angiogenesis," Tissue Engineering Part A, vol. 16, no. 7, pp. 2133-2141, 2010.

[77] C. B. Arena, C. S. Szot, P. A. Garcia, M. N. Rylander, and R. V. Davalos, "A three-dimensional in vitro tumor platform for modeling therapeutic irreversible electroporation," Biophysical Journal, vol. 103, no. 9, pp. 2033-2042, 2012.

[78] E. M. Wasson, J. W. Ivey, S. S. Verbridge, and R. V. Davalos, "The Feasibility of enhancing susceptibility of glioblastoma cells to IRE using a calcium adjuvant," Annals of Biomedical Engineering, vol. 45, no. 11, pp. 2535-2547, 2017. 